\title{
DISSOCIATION OF DAMAGE TO SPATIAL AND LUMINANCE CHANNELS IN EARLY LEBER'S HEREDITARY OPTIC NEUROPATHY MANIFESTED BY THE VISUAL EVOKED POTENTIAL
}

\author{
YUKIHIKO MASHIMA, YUTAKA IMAMURA and YOSHIHISA OGUCHI \\ Tokyo
}

\begin{abstract}
SUMMARY
Leber's hereditary optic neuropathy (LHON) is a maternally inherited disorder characterised by the acute or subacute loss of central vision, leading to severe optic atrophy. The occurrence of a demyelinating disease such as multiple sclerosis (MS) has been reported mainly in females with the mitochondrial DNA 11778 mutation. They had a poor visual outcome. To investigate the damage to the optic nerve in an early stage of LHON as compared with optic neuritis $(\mathrm{ON})$, including MS, we examined patients by testing their pattern visual evoked potential (PVEP) and flash VEP (FVEP). The mean amplitude and latency in the PVEP were reduced and delayed, respectively, in patients with LHON or ON. The mean amplitude in the FVEP was reduced in patients with LHON or ON, but the mean latency was not delayed in patients with LHON as compared with normal subjects or patients with $O N$. Luminance-related fibres may be less affected than are the spatial-vision-related fibres in patients with early LHON.
\end{abstract}

Leber's hereditary optic neuropathy (LHON) is a maternally inherited form of optic neuropathy, primarily of young males, that is characterised by the acute or subacute loss of central vision, leading to optic atrophy with cecocentral scotoma. ${ }^{1}$ The maternal inheritance may be explained by the involvement of mitochondrial DNA (mtDNA). Wallace et al. ${ }^{2}$ identified a mutation at nucleotide position (np) 11778 of mtDNA that is found exclusively in families with LHON. A number of other mtDNA mutations, especially primary mutations at positions 11778,3460

Presented at a symposium in London, 29 September 1995, in honour of Professor Geoffrey Arden.

Correspondence to: Yukihiko Mashima, MD, Department of Ophthalmology, Keio University School of Medicine, 35 Shinanomachi, Shinjuku-ku, Tokyo 160, Japan. Tel: (+81) 3-3353-1211 ext 2402. Fax: $(+81)$ 3-3359-8302. and 14484 , have been pathogenetically linked to LHON. $^{3}$

The occurrence of a demyelinating disease such as multiple sclerosis (MS) or a MS-like disease, has been reported mainly in females who carry the mitochondrial DNA 11778 mutation. ${ }^{4-6}$ These patients had a poor visual outcome. In such cases, LHON can not be distinguished from MS without molecular genetic testing, especially in the absence of a family history. However, their clinical findings in an acute stage should also differ. Recent magnetic resonance imaging (MRI) studies have demonstrated Gd-DTPA enhancement in patients with acute optic neuritis, indicating the presence of inflammation. ${ }^{7,8}$ However, few, if any, characteristic abnormalities of the optic nerve signal have been reported in patients with early LHON. ${ }^{9}$

The visual evoked potential (VEP) is a useful test for detecting MS. A delayed latency in the VEP is commonly observed in patients with demyelinating disorders. ${ }^{10.11}$ The pattern VEP (PVEP) is reported to be attentuated, delayed or desynchronised in early LHON. ${ }^{12.13}$ However, a LHON patient with the 14484 mutation was reported in whom the PVEP was significantly delayed but the critical frequency of photic driving (CFPD), that is the highest frequency of photic driving in response to repetitive flash stimulation, was normal in an early stage. ${ }^{14}$ Thus, the spatial and luminance channels of patients with LHON may be dissociated. There is no report of both PVEP and flash VEP (FVEP) testing in patients with early LHON.

To investigate the dissociation of damage between spatial and luminance channels in an early stage of LHON, we examined patients with LHON by use of the PVEP and FVEP, and compared our findings with those in patients with optic neuritis (ON), including MS. 


\section{SUBJECTS AND METHODS}

\section{Patients}

We investigated 28 Japanese patients: 12 (18 eyes) with LHON and 16 (18 eyes) with ON, including 8 patients with MS. Of the group with LHON, 9 had the 11778 mutation, 2 had the 14484 mutation and 1 had the 3460 mutation. Eleven of the 12 patients with LHON were male and one was female. Their mean age at onset of the disease was $23.5 \pm 8.1$ years (range 11-37 years). PVEP and FVEP were recorded in the 28 patients within 1 month of disease onset. Of the group with $\mathrm{ON}$ there were 10 males and 6 females, with a mean age at onset of $28.6 \pm 6.4$ years (range 13-38 years). They showed central scotomas and decreased visual acuities from 0.06 to 0.3 in the affected eye, and were treated with steroids. Their vision then recovered to 1.0 or 1.2. They do not have mtDNA LHON mutations.

Thirty normal volunteers (30 eyes) served as controls: 20 males and 10 females, mean age $30.1 \pm 6.0$ years (range $20-39$ years).

\section{Visual Evoked Potentials}

VEPs were derived monopolarly at $5 \%$ from the inion when the inion-nasion distance was $100 \%$, as

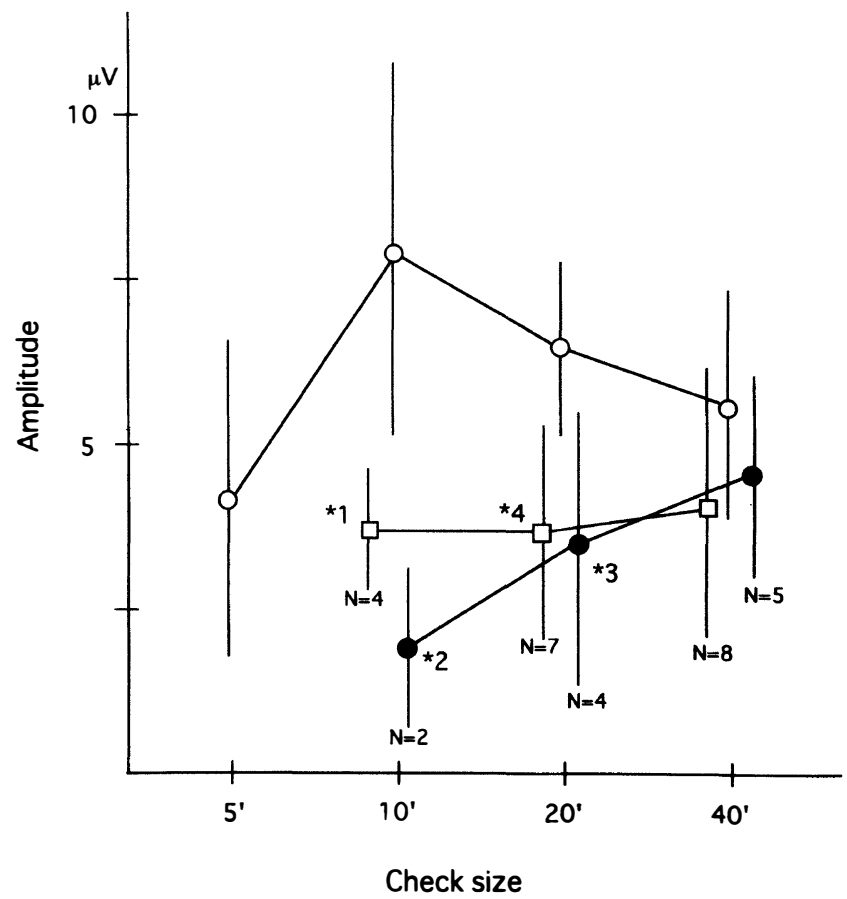

(A) described previously. ${ }^{15.16}$ Indifferent and ground electrodes ( $8 \mathrm{~mm} \mathrm{Ag}-\mathrm{AgCl}$ disc electrodes) were placed on separate earlobes. Evoked potentials were averaged 64 or 128 times. For recording the PVEP, chequerboard pattern reversal stimuli were displayed on a 20 -inch television monitor. The size of the stimulus field subtended a visual angle of $14^{\circ} \times 11^{\circ}$ observed from a distance of $160 \mathrm{~cm}$. Checks of four different sizes were used: $5^{\prime}, 10^{\prime}, 20^{\prime}$ and $40^{\prime}$. The mean luminance was $50 \mathrm{~cd} / \mathrm{m}^{2}$ with a contrast of $80 \%$. The temporal frequency of the stimulus pattern was 2 reversals per second. Latency time was

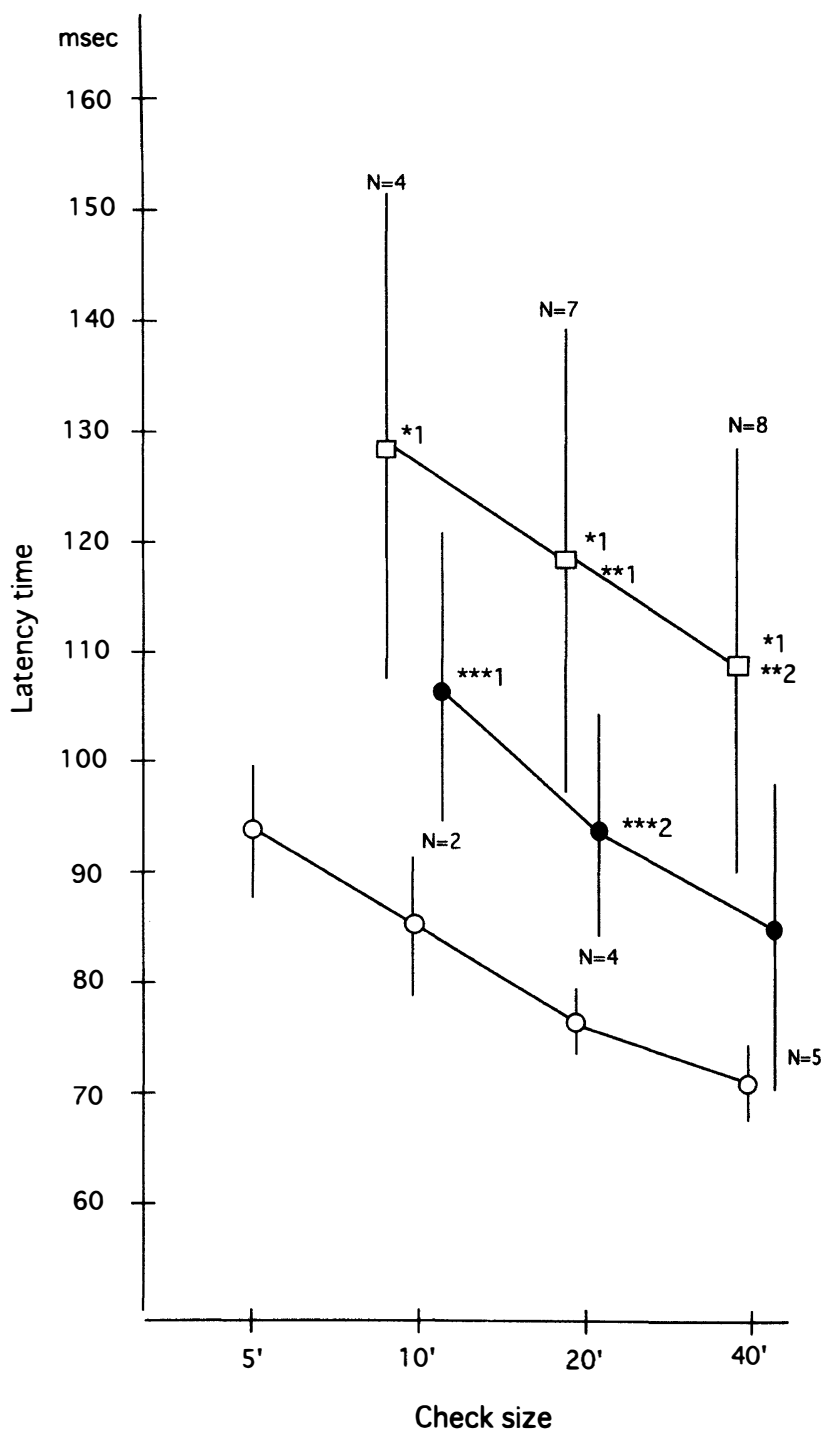

(B)

Fig. 1. Check size-amplitude curve $(A)$ and check size-latency curve $(B)$ in pattern visual evoked potentials (VEPs). Open circles and bars represent the mean $\pm S D$ in normal subjects (30 eyes). Filled circles and bars represent the mean $\pm S D$ in patients with Leber's hereditary optic neuropathy (LHON), and open squares represent the mean $\pm S D$ in patients with optic neuritis $(O N) . N$ shows the number of eyes. p values $\left({ }^{*}\right)$ represent one-factor ANOVA, Scheffé's F-test. (A) Mean amplitude was significantly reduced in both LHON and ON patients as compared with normal subjects in check sizes 10' $\left({ }^{*} 1: \mathrm{p}=0.0327\right.$, $* 2: \mathrm{p}=0.0202$, respectively), and $20^{\prime}(* 3: \mathrm{p}=0.0056, * 4: \mathrm{p}=0.0021$, respectively). There was no difference in amplitude between LHON and ON patients (p>0.05). (B) In patients with LHON, mean latency was delayed as compared with normal

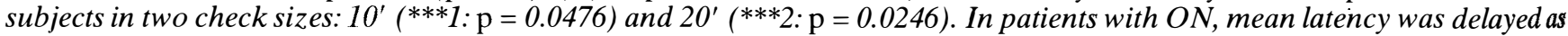
compared with normal subjects in check sizes $10^{\prime}, 20^{\prime}$ and $40^{\prime}\left({ }^{*} 1: \mathrm{p}<0.0001\right)$, and in patients with LHON in check sizes $20^{\prime}$ $(* * 1: \mathrm{p}=0.0127)$ and $40^{\prime}(* * 2: \mathrm{p}=0.0035)$. 
determined for the negative peak that appeared around $80 \mathrm{~ms}(\mathrm{~N} 80)$. The early phase reportedly represents the specific part of the visual cortex response. ${ }^{17}$ The amplitude was measured from N80 to $\mathrm{P} 100$, which appears around $100 \mathrm{~ms}$. The check size-latency curve and the check size-amplitude curve served as critical variables.

FVEPs were recorded at four different intensities of stimulus using a red filter (Fuji SC-64, Tokyo, Japan). The visual stimulus was a xenon light of 0.3 joule $(\mathrm{J})$ and of $2.0 \mathrm{~J}$. The four stimulus intensities were $0.3 \times 10^{-2} \mathrm{~J}$ (-0.2 log unit), $0.3 \times 10^{-1} \mathrm{~J}(-1.0$ $\log$ unit), $0.3 \mathrm{~J}$ (0 log unit) and $2.0 \mathrm{~J}$ (+0.8 log unit). Recording sessions were started with a $2.0 \mathrm{log}$ unit neutral density filter placed in front of the stimulus light source $(0.3 \mathrm{~J})$. The intensity-latency curve and the intensity-amplitude curve served as critical variables. ${ }^{16,18}$

\section{Statistical Analysis}

Data are presented as the mean \pm SD. Differences in amplitude or latency time in VEPs among the three groups (normal subjects, patients with LHON, patients with $\mathrm{ON}$ ) were analysed by one-factor ANOVA, Scheffé $F$-test. A value of $p<0.05$ was accepted as statistically significant.

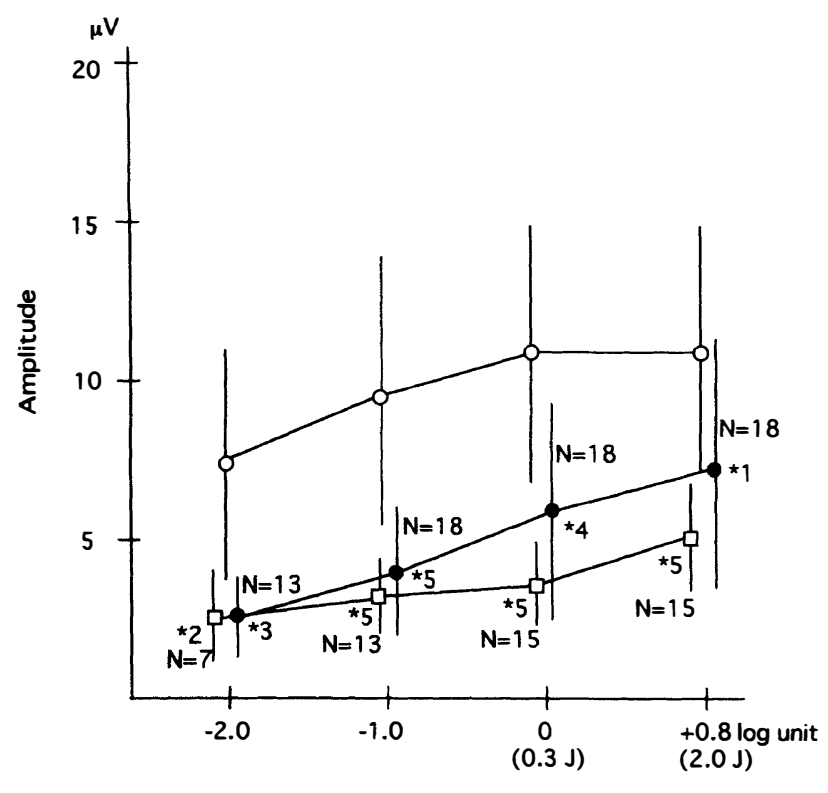

Stimulus intensity

(A)

\section{RESULTS}

The check size-amplitude curve and the check sizelatency curve are shown in Fig. 1. PVEPs were recordable in 5 of the 12 patients with LHON and in 8 of the 16 patients with ON. In normal subjects, the check size-amplitude curve represented a maximum response produced by check sizes of $10^{\prime}$ to $20^{\prime},{ }^{19}$ which suggests a band-pass function ( $\cap$-shaped curve). In patients with LHON and ON, however, the curve was linear. In LHON, as well as in ON, the mean amplitude was significantly reduced as compared with normal subjects, except for a larger check size of $40^{\prime}$ (Fig. 1A). There was no difference in amplitude between patients with LHON and ON $(p>0.05)$. The latency time in LHON as well as in ON was significantly delayed as compared with normal subjects. The delay in the latter exceeded that in the former (Fig. 1B).

The intensity-amplitude curve and the intensitylatency curve are shown in Fig. 2. FVEPs were recordable in all 12 patients with LHON and in all 16 patients with ON. Mean amplitude was significantly reduced in both patients with LHON and $\mathrm{ON}$ as compared with normal subjects (Fig. 2A). There was no difference in amplitude between patients with LHON and ON $(p>0.05)$. Mean latency time was not delayed in LHON as compared with normal subjects $(p>0.05)$, but in $\mathrm{ON}$ the mean latency time was markedly delayed as compared with normal subjects as well as with LHON patients (Fig. 2B).

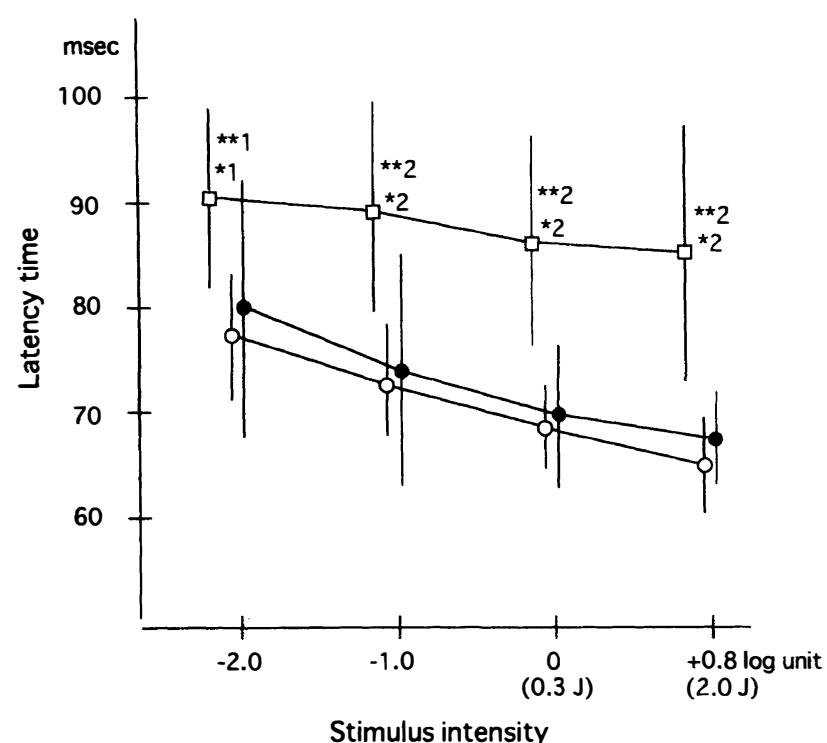

(B)

Fig. 2. Intensity-amplitude curve $(A)$ and intensity-latency curve $(B)$ in flash VEPs. Open circles and bars represent the mean $\pm S D$ in normal subjects (30 eyes). Filled circles and bars represent the mean $\pm S D$ in patients with LHON, and open squares represent the mean $\pm S D$ in patients with $O N$. N shows the number of eyes. p values $(*)$ represent one-factor $A N O V A$, Scheffés F-test. (A) Mean amplitude was significantly reduced in both LHON and ON patients as compared with normal subjects $(* 1: \mathrm{p}=0.0027, * 2: \mathrm{p}=0.0023, * 3: \mathrm{p}=0.0003, * 4: \mathrm{p}=0.0001, * 5: \mathrm{p}<0.0001)$. There was no difference in amplitude between LHON and ON patients ( $\mathrm{p}>0.05)$. (B) In patients with LHON, mean latency time was not delayed as compared with normal subjects $(\mathrm{p}>0.05)$. In patients with ON, mean latency time was markedly delayed as compared with normal subjects $\left({ }^{*} 1: \mathrm{p}=0.0006,{ }^{*} 2: \mathrm{p}<0.0001\right)$, as well as with LHON patients $\left({ }^{* *} 1: \mathrm{p}=0.242,{ }^{* *} 2: \mathrm{p}<0.0001\right)$. 
A

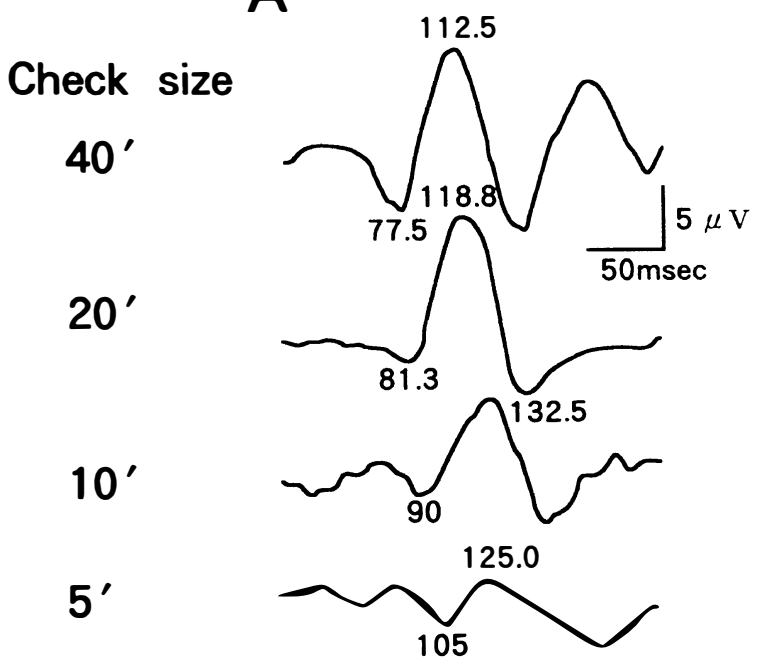

B
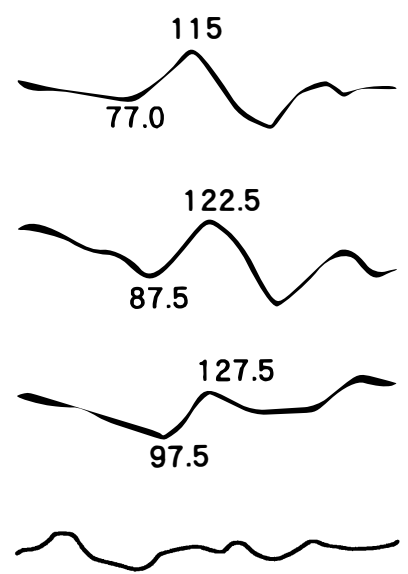

C
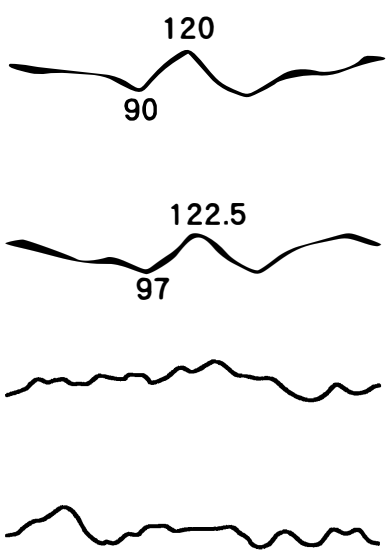

Fig. 3. Waveforms of pattern VEPS (PVEPS) from the right eye in a 23-year-old patient with LHON. In the presymptomatic stage (A), visual acuity was 25/20. Three days after the onset (B), visual acuity was 20/20. Two weeks after the onset (C), visual acuity was 20/30. Numerals represent latency time.

Fig. 3 shows PVEP waveforms in a patient with the 11778 mutation who developed LHON at the age of 23 years. VEPs were recorded in the right eye at the presymptomatic and symptomatic stages. PVEPs were not recorded in the left eye that had developed LHON 4 months earlier. In the presymptomatic stage (Fig. 3A), N80 was the upper limit of normal, P100 was slightly delayed, and the amplitudes were normal in three check sizes, except in check size $5^{\prime}$. Three days after the onset of LHON (Fig. 3B), the amplitude was markedly reduced, but N80 and P100 were unchanged. Two weeks after the onset $(\mathrm{C})$, the amplitude was markedly reduced and N80 and P100 were further delayed. PVEPs were not recordable 1 month after the onset. During onset of LHON, there was a marked reduction in amplitude, followed by a delay in latency. Fig. 4 shows the waveforms of FVEPs in the same patient as shown in Fig. 3. During an acute stage, FVEPs were slightly attenuated but not delayed, although PVEPs deteriorated rapidly.

\section{DISCUSSION}

A recent histopathological report on the atrophic stage of LHON demonstrated a striking loss of ganglion cells and nerve fibre layers in the retina. ${ }^{20}$ The optic nerve and tract appeared shrunken and atrophic with little evidence of inflammation. LHON supposedly affects primarily the retinal ganglion cells and their axons.

\section{A}

\section{Stimulus intensity}

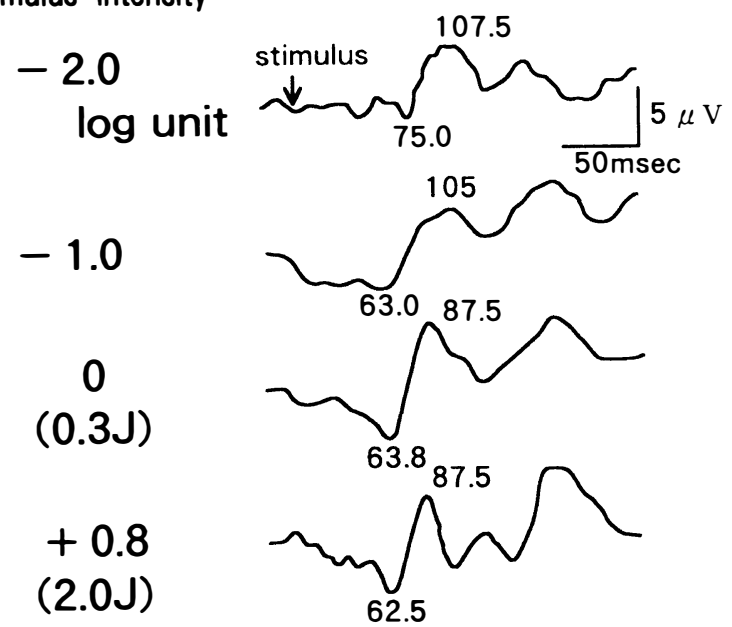

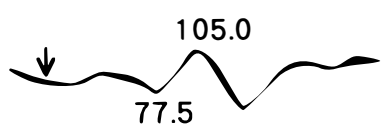
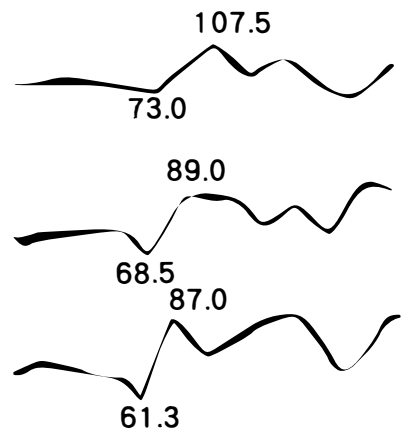

C

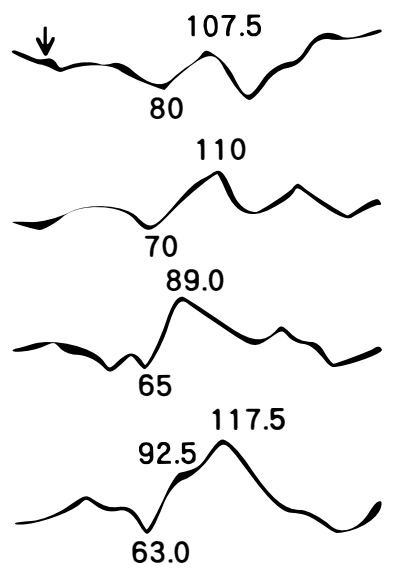

Fig. 4. Waveforms of flash VEPs (FVEPs) from the right eye in the same patient as shown in Fig. 3: presymptomatic stage $(A), 3$ days after the onset $(B)$ and 2 weeks after the onset $(C)$. Numerals represent latency time. Stimulus delay was 20 ms after a trigger signal (vertical arrows). 
Patients with MS reportedly show no pathological changes in the retina, but do show plaques and periphlebitis in the optic nerve, chiasm and tract. ${ }^{21}$ While the axonal myelin sheaths undergo destruction in MS, the axons are often well preserved. ${ }^{22,23}$ LHON differs histopathologically from MS.

Few studies of VEP performed at an early stage of LHON have been reported. Dorfman et al. ${ }^{12}$ studied PVEP in two brothers with early LHON. One month after the onset, the latency of VEP was normal. Then, the response developed prolonged latency and reduced amplitude with the waveform developing a bifid positivity in a $\mathrm{W}$ configuration. The pattern size used was a check of $1^{\circ} 50^{\prime}$ of arc. Carroll and Mastaglia ${ }^{13}$ also found that PVEP in patients with LHON was less delayed in P100 latency, but showed a greater reduction in amplitude, and was disorganised. They speculated that these findings implied a more severe disturbance of conduction in the optic nerve, consistent with a combination of demyelination and the loss of nerve fibres. ${ }^{13}$ The pattern size used was a check of $12^{\prime}$ of arc. PVEPs were normal in most of the asymptomatic individuals in a LHON family, but were absent in the symptomatic individuals. ${ }^{24}$

We investigated optic nerve conduction using the PVEP and FVEP in 12 patients with LHON and in 16 patients with $\mathrm{ON}$ within 1 month after the onset. We recorded the PVEP and FVEP from a patient with LHON in both presymptomatic and symptomatic stages, and found the same result as had Carroll and Mastaglia ${ }^{13}$ : a marked reduction in amplitude followed by latency delay in PVEP. Subsequently, the PVEP became non-recordable. However, FVEPs were less affected during such periods. In this study, the most striking manifestations of the effects on the VEP in LHON were those apparent on the FVEP. PVEPs were reduced and delayed, or not recordable in patients with LHON or ON. However, in the patients with LHON, the FVEPs were reduced but not delayed. This finding may suggest that most of the fibres in the luminance channels were less affected by LHON than were fibres in the spatial frequency channels. Latency time (N80) was less affected in LHON than in ON. In patients with demyelinating $\mathrm{ON}$, a delay of latency was marked for both FVEP $^{10}$ and PVEP. ${ }^{11}$ The characteristics of FVEPs in the patients with LHON resemble those of patients with toxic neuropathy ${ }^{10}$ and with compression of the optic nerve. ${ }^{10.18}$ The early stage of LHON differs electrophysiologically from that of $\mathrm{ON}$.

The patient with early LHON exhibits central or centrocecal scotomas. The optic disc is swollen and hyperaemic. Retinal arterioles on and around the disc are dilated, tortuous and telangiectatic. Fluorescein angiography shows no leakage of dye from the telangiectatic vessels. The nerve fibre layer is also swollen. Several weeks after the onset, there is a reduction in size of the telangiectatic arterioles on the temporal side of the disc accompanied by nerve fibre layer 'dropout' (axonal loss) in a narrow channel in the papillomacular bundle. ${ }^{25}$ Consequently, optic atrophy with nerve fibre layer defect is most pronounced in the papillomacular bundle. Nikoskelainen et al. ${ }^{25}$ suggested that LHON causes intraocular, not retrobulbar neuropathy. Thus, the finding in patients with early LHON that the VEP was less delayed in latency but more markedly reduced in amplitude may be associated with selective axonal loss in the papillomacular bundle in the retina.

The critical frequency of photic driving (CFPD) was reportedly normal in a patient with LHON who showed a significant delay in the VEP response to pattern stimuli for a check of $56^{\prime}$ of arc. ${ }^{14}$ Patients with demyelinating disease typically exhibit diminished CFPD values. ${ }^{26}$ Another report found that latency of the flash-evoked blink reflex was normal in 10 of 11 patients with LHON, while 12 of 15 patients with other forms of ON showed delayed or extinguished response. ${ }^{27}$ The direct pupillary response to light was reported to be preserved in LHON, as compared with ON. ${ }^{28}$ The authors ${ }^{28}$ speculated that in LHON, W cells functioning as a luminance detector and providing input to the pupillary light response were relatively intact, in contrast to an impairment of the $X$ and $Y$ cells. In an acute stage of LHON, luminance-related fibres or pupillary-reflexrelated fibres may be less affected than are the spatial-frequency-related fibres, whereas in patients with $\mathrm{ON}$ all these types of fibres are damaged by inflammation or demyelination of the optic nerve. ${ }^{26-29}$

Key words: Leber's hereditary optic neuropathy, Multiple sclerosis, Visual evoked potential, Luminance, Latency.

\section{REFERENCES}

1. Miller N. Hereditary optic neuropathies. In: Walsh and Hoyt's clinical neuro-ophthalmology. 4th ed. vol 1. Baltimore: Williams and Wilkins, 1982:311-7.

2. Wallace DC, Singh G, Lott MT, Hodge JA, Schurr TG, Lezza AMS, Elsas LJ II, Nikoskelainen EK. Mitochondrial DNA mutation associated with Leber's hereditary optic neuropathy. Science 1988;242:1427-30.

3. Newman NJ. Leber's hereditary optic neuropathy: new genetic considerations. Arch Neurol 1993;50:540-58.

4. Harding AE, Sweeney MG, Miller DH, Mumford CJ, Kellar-Wood H, Menard D, McDonald WI, Compston AS. Occurrence of a multiple sclerosis-like illness in women who have a Leber's hereditary optic neuropathy mitochondrial DNA mutation. Brain 1992; 115:979-89.

5. Flanigan KM, Johns DR. Association of the 11778 mitochondrial DNA mutation and demyelinating disease. Neurology 1993;43:2720-2. 
6. Kellar-Wood H, Robertson N, Govan GG, Compston DAS, Harding AE. Leber's hereditary optic neuropathy mitochondrial DNA mutations in multiple sclerosis. Ann Neurol 1994;36:109-12.

7. Guy J, Mancuso A, Quisling RG, Beck R, Moster M. Gadolinium-DTPA-enhanced magnetic resonance imaging in optic neuropathies. Ophthalmology 1990;97:592-600.

8. Youl BD, Turano G, Miller DH, Towell AD, MacManus DG, Moore SG, Jones SJ, Barrett G, Kendall BE, Moseley IF, Tofts PS, Halliday AM, McDonald WI. The pathophysiology of acute optic neuritis: an association of gadolinium leakage with clinical and electrophysiological deficits. Brain 1991;114:2437-50.

9. Dotti MT, Caputo N, Signorini E, Federico A. Magnetic resonance imaging findings in Leber's hereditary optic neuropathy. Eur Neurol 1992;32:17-9.

10. Babel J, Stangos N, Korol S, Spiritus M. The visual evoked response (VER). In: Ocular electrophysiology. Stuttgart: Thieme, 1977:95-128.

11. Halliday AM. The visual evoked potential in the investigation of diseases of the optic nerve. In: Halliday AM, editor. Evoked potentials in clinical testing. New York: Churchill Livingstone, 1982:187-234.

12. Dorfman LJ, Nikoskelainen E, Rosenthal AR, Sogg RL. Visual evoked potentials in Leber's hereditary optic neuropathy. Ann Neurol 1977;1:565-8.

13. Carroll WM, Mastaglia FL. Leber's optic neuropathy: a clinical and visual evoked potential study of affected and asymptomatic members of a six generation family. Brain 1979;102:559-80.

14. Kothe AC, Lovasik JV, Pace R, Hrynchak PK, Flanagan JG. Visual and neural function in Leber's optic neuropathy. Optom Vis Sci 1990;67:138-47.

15. Katsumi O, Oguchi Y, Uemura Y. Studies on 'meridional amblyopia' by VECP after pattern reversal stimulation. Ophthalmic Pediatr Genet 1982;1: 113-24.

16. Mashima Y, Matsuhashi M, Oguchi Y. Simultaneous recording of visual evoked potentials and electroretinograms in patients with unilateral opaque media. Acta Ophthalmol (Copenh) 1988;66:47-53.
17. Ciganec L. The EEG response (evoked potential) to light stimulus in man. Electroencephalogr Clin Neurophysiol 1961;13:165-72.

18. Mashima Y, Oguchi Y. Visual evoked potential in the management of pituitary tumor during pregnancy. Doc Ophthalmol 1987;65:57-64.

19. Spekreijse H, Van der Tweel LH, Zuidema T. Contrast evoked responses in man. Vision Res 1973;13: 1577-601.

20. Sadun AA, Kashima Y, Wurdeman AE, Dao J, Heller $\mathrm{K}$, Sherman J. Morphological findings in the visual system in a case of Leber's hereditary optic neuropathy. Clin Neurosci 1994;2:165-72.

21. Mogensen PH. Histopathology of anterior parts of the optic pathway in patients with multiple sclerose. Acta Ophthalmol (Copenh) 1990;68:218-20.

22. McDonald WI. The pathogenesis of optic neuritis. In: Hess RF, Plant GT, editors. Optic neuritis. Cambridge: Cambridge University Press, 1986:42-50.

23. Klintworth GK. Ocular involvement in disorders of the nervous system. In: Garner A. Klintworth GK, editors. Pathobiology of ocular disease. New York: Dekker, 1994:1711-34.

24. Livingstone IR, Mastaglia FL, Howe JW, Aherne GES Leber's optic neuropathy: clinical and visual evoked response studies in asymptomatic and symptomatic members of a 4-generation family. $\mathrm{Br} \mathrm{J}$ Ophthalmol 1980;64:751-7.

25. Nikoskelainen E, Hoyt WF, Nummelin K. Ophthalmoscopic findings in Leber's hereditary optic neuropathy. II. The fundus findings in the affected family members. Arch Ophthalmol 1983;101:1059-68.

26. Cohen SN, Syndulko K, Tourtellotte WW, Potvin AR. Critical frequency of photic driving in the diagnosis of multiple sclerosis. Arch Neurol 1980;37:80-3.

27. Nakamura M, Sekiya Y, Yamamoto M. Preservation of photic blink reflex in Leber's hereditary optic neuropathy. Invest Ophthalmol Vis Sci 1996;37:2736-43.

28. Wakakura M, Yokoe J. Evidence for preserved direct pupillary light response in Leber's hereditary optic neuropathy. Br J Ophthalmol 1995;79:442-6.

29. Bos JE, Fock JM, Reulen JPH, Van Lith GHM. Pupil constriction and visual evoked potential latency in optic neuritis. Neuro-ophthalmology 1990;10:187-96. 